\title{
DNA chip-based expression profile analysis indicates involvement of the phosphatidylinositol signaling pathway in multiple plant responses to hormone and abiotic treatments
}

\author{
Wen Hui LIN, Rui YE, Hui MA, Zhi Hong XU, Hong Wei XUE*
}

National Laboratory of Plant Molecular Genetics, Institute of Plant Physiology and Ecology, Shanghai Institutes for Biological Sciences (SIBS), Chinese Academy of Sciences; and Partner Group of Max-Planck-Institute of Molecular Plant Physiology (MPI-MP) on "Plant Molecular Physiology and Signal Transduction", 300 Fenglin Road, 200032 Shanghai, China

\begin{abstract}
The phosphatidylinositol (PI) metabolic pathway is considered critical in plant responses to many environmental factors, and previous studies have indicated the involvement of multiple PI-related gene families during cellular responses. Through a detailed analysis of the Arabidopsis thaliana genome, 82 polypeptides were identified as being involved in PI signaling. These could be grouped into different families including PI synthases (PIS), PI-phosphate kinases (PIPK), phospholipases (PL), inositol polyphosphate phosphatases (IPPase), inositol polyphosphate kinases (IPK), PI transfer proteins and putative inositol polyphosphate receptors. The presence of more than 10 isoforms of PIPK, PLC, PLD and IPPase suggested that these genes might be differentially expressed during plant cellular responses or growth and development. Accordingly, DNA chip technology was employed to study the expression patterns of various isoforms. In total, 79 mRNA clones were amplified and used for DNA chip generation. Expression profile analysis was performed using samples that represented multiple tissues or cellular responses. Tested samples included normal leaf, stem and flower tissues, and leaves from plants treated with various hormones (auxin, cytokinin, gibberellin, abscisic acid and brassinosteroid) or environmental factors (temperature, calcium, sodium, drought, salicylic acid and jasmonic acid). Results showed that many PI pathway-related genes were differentially expressed under these experimental conditions. In particular, the different isoforms of each family were specifically expressed in many cases, suggesting their involvement in tissue specificity and cellular responses to environmental conditions. This work provides a starting point for functional studies of the relevant PI-related proteins and may help shed light onto the role of PI pathways in development and cellular responses.
\end{abstract}

Keywords: phosphatidylinositol, signal transduction, DNA chip, expression profile.

\section{INTRODUCTION}

Although they were first described in the 1950s, it was only recently that researchers recognized the critical importance of phospholipids and the related phosphatidylinositol (PI) signaling pathway in higher plants comparing to the relatively detailed studies in animal cells. In particular, the second messengers inositol 1,4,5trisphosphate $\left[\operatorname{Ins}(1,4,5) \mathrm{P}_{3}\right]$ and diacylglycerol (DAG) were recently demonstrated to be critical in signal transduction processes such as gene expression, hormone function, cell differentiation, transduction of intracellular

\footnotetext{
* Correspondence author: Hong Wei XUE

Tel: +86-21-64042090 ext 4411,

Fax: +86-21-64042385,

E-mail: hwxue@sibs.ac.cn
}

signaling molecules, control of cell responses to environmental factors, regulation of ion-channel gating, energy metabolism and rearrangement of cytoskeleton[1]. In addition, it has been shown that deficiency of these proteins results in exceptional physiological phenomena such as changed cell response to hormone treatments, and some abiotic stresses, which perhaps even involves the interaction with other signaling pathways such as the calciumrelated signaling network $[2,3]$.

To begin the PI metabolic pathway, PI synthase (PIS) synthesizes PI from membrane-bound CDP-diglycerol (CDP-DG) and free cytoplasmic inositol. Sequential phosphorylations at the 4- and 5- positions by PI4-kinase (PI4K) and PI-phosphate 5-kinase (PIPK) result in the production of $\mathrm{PI}(4,5) \mathrm{P}_{2}[4]$, which is the substrate of phospholipase C (PLC) and the direct precursor of two important second messenger molecules, $\operatorname{Ins}(1,4,5) \mathrm{P}_{3}$ and diacylglycerol 
(DAG), which can stimulate $\mathrm{Ca}^{2+}$ release from internal calcium stores and activate related protein kinases for regulation of downstream pathways or cellular responses. In a tightly regulated manner, these second messengers may be recycled by further phosphorylation of $\operatorname{Ins}(1,4,5) \mathrm{P}_{3}$ to $\operatorname{Ins}(1,3,4,5) \mathrm{P}_{4}$ by Ins $(1,4,5) \mathrm{P}_{3}$ 3-kinase (IP3K), or dephosphorylation to $\operatorname{Ins}(1,4) \mathrm{P}_{2}$ by inositol polyphosphate 5-phosphatase (IPPase). To date, most molecular characterizations, expression pattern analyses and biochemical studies have focused on key PI pathway-related proteins including PIS [5], PI4K[6-8], PIP5K[9-13], PLC [14,15], PLD [16], PLA 1 [17], PLA 2 [18, 19], PI 3-kinase (PI3K) [20], IPPase [21] and inositol 1, 3, 4-trisphosphate 5/6-kinase $\left(\mathrm{IP}_{3} 5 / 6 \mathrm{~K}\right)[22]$. In contrast, little attention has been paid to other proteins such as inositol 1, 4, 5trisphosphate receptor $\left(\mathrm{IP}_{3} \mathrm{R}\right)$ and PI transfer protein (PITP), and there are many other signal molecules yet to be investigated within this pathway. These signal molecules come from Ins $(1,4,5) \mathrm{P}_{3}$ or phosphoinositides and may act as additional second messenger molecules. Examples of these are $\mathrm{PI}(3,4,5) \mathrm{P}_{3}$ (phosphorylated by $\left.\mathrm{PI} 3 \mathrm{~K}\right), \operatorname{Ins}(1,3$, $4,5) \mathrm{P}_{4}$ (phosphorylated by IP3K), Ins $(1,3,4) \mathrm{P}_{3}$ and the putative $\mathrm{IP}_{3} \mathrm{R}$ and PITPs, many or all of which may important for proper signaling.

Many members of the PI pathway are located in or near the membrane, though a few are nuclear[23]. The diversity and possible function of the PI pathway has been extensively studied in mammals, including humans [24, 25], though few studies have investigated the pathway in plants. Little is known about the physiological functions and components of the overall PI pathway in plants, though the genes for PIS, PI4K, PIPK, PLC and PLD have been isolated and characterized from Arabidopis thaliana, Oryza sativa and Glycine max. Additionally, the identification of multiple isoforms of proteins such as PIPK[26], PLC, PLD [27] and IPPase (Lin et al., in preparation), as well as their differential expression patterns suggests the presence and regulation of a complex PI pathway within plant tissues.

The completed sequencing of the $A$. thaliana genome allowed the first genome-wide screens for relevant genes [28], and the recent development of cDNA chip technology made possible the large-scale analysis of light control-related genes[29], specific gene expression in developing seeds [30], drought- and wound-related genes[31, 32], rice ABA-responsive genes[33], and the relative effects of mutant vs. wild-type gene expression[34].

However, few recent studies have focused on expression profile analysis of a specific metabolic and signaling pathway, although relatively detailed structural analysis has been performed on PLD[27], PIPK and PLC [26] and IPPase (Lin et al., in preparation). Here, we used DNA chip technology to investigate the expression profiles of
A. thaliana genes involved in the PI pathway. In addition, we investigated differential expression of these genes in various tissues and under various stresses or conditions, allowing us to develop a greater understanding of the importance of the PI pathway. This is the first report on the expression profile analysis of a single plant metabolic pathway, and the results will aid researchers by identifying important proteins for further functional studies and hinting at their interrelation in the PI pathway.

\section{MATERIALS AND METHODS}

\section{Enzymes and chemicals}

PCR primers were synthesized by TibMolBio (Berlin, Germany). 24-epibrassinolide (24-eBL, \#E-1641), abscisic acid (ABA, \#A1049), indole-3-acetic acid (IAA, \#I2886), gibberellic acid ( $\left.\mathrm{GA}_{3}, \# \mathrm{G} 7645\right)$, kinetin (KT, \#K0753) and salicylic acid (SA, \#S7401) were obtained from Sigma (USA). Methyl jasmonate (MeJA, \#39270-7) and mannitol (\#24018-4) were obtained from Aldrich (USA). $\mathrm{CaCl}_{2}$ and $\mathrm{NaCl}$ were obtained from Sangon Company (Shanghai). All other reagents used were standard analytical or electrophoresis grade.

\section{Analysis of PI-related genes in A. thaliana genome}

To analyze the PI-related genes on a genome-wide level, key words such as phosphatidylinositol, phospholipase, inositol, phosphatase, trisphosphate, kinase and inositol 1, 4, 5-trisphosphate receptor were used to search the $A$. thaliana genome database contained within the National Center of Biotechnological Information (NCBI, http:// www.ncbi.nlm.nih.gov/entrez). Additionally, key words including inositol, phosphatidylinositol, phospholipase and receptor, as well as isolated cDNA sequences encoding PI synthase, PI kinase, PIP kinase, phospholipase, were used as baits to search the database. The resulting genomic clones and predicted mRNA sequences were further used for sequence analysis and design of PCR primers.

\section{Primer design and amplification of DNA fragments}

Primers were designed from the deduced mRNA sequences identified in the above screen or the isolated ones. Sequence comparison analysis and previous studies indicated that most of the PI pathwayrelated genes were most highly conserved at the C-terminus. Therefore, we designed PCR primers to amplify 600-800 bp Nterminal fragments (spanning the first 1-3 exons), so as to avoid nonspecific amplification (Tab 1). The primers were used for amplification of $A$. thaliana genomic DNA, and the PCR products were checked on agarose gel via electrophoresis. A total of 93 DNA fragments were successfully amplified, purified and spotted on glassbased chips. Because some genes were represented multiple times (with different accession numbers) within the database, some clones were multiply represented on the cDNA chips.

\section{Plant materials and treatment with hormones and compounds}

To study the expression profiles of PI-related genes under different conditions, multihormones and stress treatments were performed so as to illustrate their differential expression patterns. Seeds of $A$. thaliana (Columbia) were surface sterilized and germinated on PNS 
culture medium with vernalization for 2 days. Germinated seedlings were grown in a dark culture room for 1 day to improve the germination and then further grown under $16 \mathrm{~h}$ light $\left(20^{\circ} \mathrm{C}\right)$ and $8 \mathrm{~h}$ dark $\left(20^{\circ} \mathrm{C}\right)$ conditions for another 5 days. After budding, shoots were transferred to pots and grown in the above conditions in a phytotron (70-80\% of humidity). After 4 weeks, leaves, stems and flowers of untreated plants, and leaves of treated plants were harvested and used for RNA extraction. For investigation of hormonal and stress responses, plants were given IAA $(10 \mu M)$, 24-epi-brassinolide (24eBL, $10 \mu M)$, ABA $(10 \mu M)$, KT $(10 \mu M), \mathrm{GA}_{3}(10 \mu M)$, SA (salicylic acid, $200 \mu M$ ), MeJA (Methyl Jasmonate, $100 \mu M)$ ), mannitol (250 $\mu M), \mathrm{CaCl}_{2}(5 \mathrm{~m} M)$ or $\mathrm{NaCl}(200 \mathrm{~m} M)$ for $4 \mathrm{hs}$. For investigation of responses to environmental stresses, plants were treated with cold $\left(4^{\circ} \mathrm{C}\right.$, for $\left.16 \mathrm{~h}\right)$ or drought $\left(55^{\circ} \mathrm{C}\right.$, for $\left.1 \mathrm{~h}\right)$. Selected tissues and treated materials were collected and frozen at $-70^{\circ} \mathrm{C}$ prior to use.

\section{DNA chip preparation}

Amplified PCR products were isopropanol precipitated, examined on an agarose gel to ensure quality, and dried (SAVANT SPEEDVAC PLUS, SC 210A, Thermo Life Science). Samples were then dissolved in 3X SSC, and spotted four times each onto silicificated slides (Biostar, Shanghai, China) with an Omnigrid Arrayer (Gene Machine, CA) fitted with 16 Major Precision split pins. Slides were hybridized for 2 hours in $70 \%$ humidity, dried for 0.5 hour at room temperature and UV cross-linked $(65 \mathrm{mj} / \mathrm{cm})$. This was followed by soaking in room temperature $0.2 \%$ sodium dodecyl sulfate (SDS) for 10 min, distilled $\mathrm{H}_{2} \mathrm{O}$ for $10 \mathrm{~min}$, and $0.2 \%$ sodium borohydride (NaBH4) for $10 \mathrm{~min}$. The slides were then dried prior to use. To facilitate the evaluation and analysis of chip, mock buffer and 10 house-keeping gene of human and mouse were spotted on the chip as negative controls.

\section{RNA purification, probe labeling and hybridization}

Tissue samples and treated $A$. thaliana leaves were ground into a fine powder in a $10 \mathrm{~cm}$ ceramic mortar (RNase-free) and homogenized in Trizol solution (Biostar, Shanghai, China). After centrifugation, the supernatant was extracted with an equal volume of chloroform, and the aqueous phase was precipitated with an equal volume of isopropanol at $4{ }^{\circ} \mathrm{C}$. The resultant RNA pellet was dissolved in Milli-Q $\mathrm{H}_{2} \mathrm{O}$. The fluorescent cDNA probes were prepared through reverse transcription with Cy3- or Cry5-deoxy UTP (Amersham Phamacia Biotech, Piscataway, NJ) as follows: $5 \mu \mathrm{g}$ of oligo $(\mathrm{dT})_{18}$ (or antisense primers at concentrations identical to those used for PCR) and $50 \mu \mathrm{g}$ of total RNA were incubated at $70^{\circ} \mathrm{C}$ for 10 $\mathrm{min}$, and then chilled on ice. The reaction was carried out in a mixture containing dNTPs (200 $\mu M$ dATP, dCTP, dGTP, $60 \mu M$ dTTP, and $60 \mu M$ Cy3- or Cry5-dUTP), $2 \mu \mathrm{L}$ of Superscript II reverse transcriptase (Invitrogen) and $1 \mathrm{X}$ reaction buffer for $2 \mathrm{hs}$ at $42^{\circ} \mathrm{C}$. Then the RNA was hydrolyzed by addition of $4 \mu \mathrm{L}$ of $2.5 \mathrm{M} \mathrm{NaOH}$ and incubation at $65^{\circ} \mathrm{C}$ for $10 \mathrm{~min}$, and neutralized with $4 \mu \mathrm{L}$ of $2.5 \mathrm{M}$ $\mathrm{HCl}$. For the most part, the control samples (RNA extracted from untreated materials) were labeled with Cy3-dUTP and treated RNAs were labeled with Cy5-dUTP. After quantitation of fluorescence, the labeled probes were mixed and diluted in $500 \mu \mathrm{L}$ TE solution and concentrated to $10 \mu \mathrm{L}$ with a Microcon YM-30 filter (Millipore, Bedford, MA).

Labeled probes were purified and dissolved in $20 \mu \mathrm{L}$ of hybridization solution ( $5 \mathrm{x} \mathrm{SSC}, 0.75 \mathrm{M} \mathrm{NaCl}$ and $0.075 \mathrm{M}$ sodium citrate) with $0.4 \%$ SDS and $50 \%$ formamide. DNA chips were pre-hybridized with hybridization solution containing $0.5 \mathrm{mg} / \mathrm{ml}$ denatured salmon sperm DNA at $42^{\circ} \mathrm{C}$ for $6 \mathrm{~h}$. Fluorescent probes were denatured at $95^{\circ} \mathrm{C}$ for $5 \mathrm{~min}$ and applied to the pre-hybridized chips under a cover glass. Chips were hybridized at $42^{\circ} \mathrm{C}$ for $15-17 \mathrm{hs}$. After hybridization, the chips were washed at $60^{\circ} \mathrm{C}$ for $10 \mathrm{~min}$ each in solutions of $2 \mathrm{XSSC}$ and $0.2 \% \mathrm{SDS}, 0.1 \mathrm{X}$ SSC and $0.2 \% \mathrm{SDS}$ and $0.1 \mathrm{x} \mathrm{SSC}$, then dried at room temperature.

\section{Chip scanning and data analysis}

Hybridized chips were scanned with a ScanArray 4000 (GSI Lumonics, Billerica, MA) at 532 and $653 \mathrm{~nm}$ to detect emission from $\mathrm{Cy} 3$ and $\mathrm{Cy} 5$, respectively. The resulting images were analyzed using GenePix Pro 3 software (Axon Instruments Inc., USA). The intensities of each spot at the two wavelengths represented the quantity of Cy3-dUTP and Cy5-dUTP, and the ratios of Cy3 to Cy5 were computed using the GenePix Pro 3 median of ratio method. Overall intensities were normalized using the corresponding GenePix default normalization factor. All spots flagged 'Bad' or 'Not Found' by GenePix software were removed from the final data set. Genes were identified as differentially expressed if the absolute value of the natural logarithm of the ratios was $>0.69$, which indicated at least one time induction or suppression. Only genes with raw intensity values for both Cy 3 and $\mathrm{Cy} 5$ of $>200$ counts were chosen for further analysis.

Cluster analysis was performed with the help of Gene Cluster Program (http://rana.stanford.edu/software) to study the expression pattern of PI-related genes in vegetative tissues (flower, root and stem), following hormone treatment and stress conditions.

\section{RESULTS}

\section{Multiple PI pathway-related gene families are present in the $\boldsymbol{A}$. thaliana genome}

Previous studies have indicated that many proteins including PIS, PI4K, PIP5K, PLC, PLD, IPPase and IP3K are involved in the PI pathway, and that others, such as PI3K, IP3R and PITP, are implicated in the pathway. Searching against the $A$. thaliana genome database using different key words including inositol, phosphatidylinositol, and phospholipase receptor, as well as using isolated cDNA sequences as baits for homologous search, identified 82 PI pathway-related isoforms scattered throughout the genome, including 3 PISs, 4 PI4Ks, 10 PIPKs, 17 PLCs, 10 PLDs, 5 PLA $_{2}$ s, 8 putative PLs, 4 IPKs, 1 PI3K, 2 IPRs, 15 IPPases, and 3 PITPs (Tab 1). Sequence analysis revealed that within each family, the isoforms were highly homologous at their C-termini, in which contain catalytic regions [37]. The exception to this was the PIPKs and IPPases (structural subfamily I), which contained conserved $\mathrm{N}$-terminal MORN regions and WD- 40 repeats, respectively. DNA amplification, chip preparation, hybridization
and data analysis 
Tab 1 The Arabidopsis thaliana genome contains members of multiple PI pathway-related gene families. Genes were identified by BLAST search against the Arabidopsis database. Gene IDs and their chromosomal localizations are indicated. Primers used for DNA or cDNA amplification are shown. PIS, PI synthase; PI4K, PI 4-kinase; PIPK, PI phosphate kinase; PL, phospholipase; IPK, inositol polyphosphate kinase; PI3K, PI 3-kinase; IPR, IP $_{3}$ receptor; IPPase, inositol polyphosphate phosphatase.

\begin{tabular}{|c|c|c|c|c|c|}
\hline & Name & Gene ID & Chr. & Forward primer $\left(5^{\prime}-3^{\prime}\right)$ & Reverse primer $\left(5^{\prime}-3^{\prime}\right)$ \\
\hline \multirow[t]{5}{*}{ PIS } & PIS1 & AT1G68000 & 1 & AAGGAGAGACCTAGACCTGAGAAG & GCTTCCTACATATTCTAGTCATGGC \\
\hline & & & & AGGAGAGACCTAGACCTGAGAA & GGCTTCTGCTGCTTCTCTATA \\
\hline & PIS2 & AT4G38570 & 4 & CGACGTTGTCAGTCTACCTCTACATTC & CTGTGCTGACTCTGTAACCATGC \\
\hline & & & & GACGTTGTCAGTCTACCTCTACATTC & GAGTTGTGTGTTACGTTCAATCG \\
\hline & PIS3 & AT1G51040 & 1 & AGGCTAAGTCCATCAACAAAGCAC & ATCGAGTACATCATCCATCACCTG \\
\hline \multirow[t]{5}{*}{ PI4K } & PI4K $\beta 1$ & AT5G64070 & 5 & СтTTCTATCTTTGGTCCGAGGAGAC & GCATCTCTGACTTTAGGACTTGGG \\
\hline & & & & ATGCCGATGGGACGCTTTCTA & CCTCTGAGTTAGGTATTGGTTCA \\
\hline & $\mathrm{PI} 4 \mathrm{~K} \alpha 1$ & AT1G49340 & 1 & CATGTTACGGATGTGTCTGCATAG & TACCACCGTATCTGTTTCATCCAG \\
\hline & $\mathrm{PI} 4 \mathrm{~K} \alpha 2$ & AT1G51040 & 1 & GAGAGATTGAATGAGCTTTGCGAC & TATTTCCTCAACAGACTCACCCTG \\
\hline & $\mathrm{PI} 4 \mathrm{~K} \beta 2$ & AT5G09350 & 5 & GATTCGATTGAGTCTCCTCGTGAG & ACATTCCTCGTCTTCTTTGTCAGC \\
\hline \multirow[t]{11}{*}{ PIPK } & PIP5K1 & AT1G21980 & 1 & GTACGAAGGAGATTGGAAACGAG & GCССТTTAGAAATAGTCTGTCCAGG \\
\hline & & & & ACTTGGAGTGATGGGAGTTCTTG & CTTTCCAGGTGAAGATAGTTCGC \\
\hline & PIP5K2 & AT1G77740 & 1 & AAGGATGGGTGTATGTACGAAGG & CTCTCCAGGTTTCTTAGCCTCAC \\
\hline & PIP5K3 & AT2G26420 & 2 & GGCTGCCGAGATTAGAATAGTAGA & TACATGATCACACACGCCAGTATC \\
\hline & PIP5K4 & AT3G56960 & 3 & GTTAATGGCCACTGATCAACCAC & GTTGAGAAGCTATTGCTGCTGAG \\
\hline & PIP5K5 & AT2G41210 & 2 & TTTGGAACAGTATCGGTAGCTCC & CATTTCCTCTGGATCCTTAGACC \\
\hline & PIP5K6 & AT3G07960 & 3 & GATGAATCTGAGACATGGGCAAG & CCAAGCTGCAAGTTAAGCATGAG \\
\hline & PIP5K8 & AT1G60890 & 1 & GACCGTAAGAAAGACCCAACAAG & ACCAACGTAGAAACTCCCATCAG \\
\hline & PIP5K9 & AT3G09920 & 3 & CTGGTCAACTCAAAGGAACACTTC & CTCAGAAAGACTTCGTGATACAGC \\
\hline & PIP5K10 & AT1G01460 & 1 & TCCTCCTGGAACCATTACTGAGT & GTCTGGCCAAAGAATCCACATAG \\
\hline & PIP5K11 & $\mathrm{P} 5 \mathrm{~K} 2$ & 2 & CGCGTTTCATGTGTCTGACTATC & AAGATCCGTTCCTCTGGTCCTAA \\
\hline \multirow[t]{21}{*}{ PLC } & PLC1 & AT4G38690 & 4 & CGCGTTTCATGTGTCTGACTATC & AAGATCCGTTCCTCTGGTCCTAA \\
\hline & & & & CGCGTTTCATGTGTCTGACTATC & AAGATCCGTTCCTCTGGTCCTAA \\
\hline & & & & TCCATCACCAGAAGAACTTAAGGG & CAACATGAGGATCGTAGTTTGACG \\
\hline & PLC2 & AT3G08510 & 3 & CGCGTTTCATGTGTCTGACTATC & AAGATCCGTTCCTCTGGTCCTAA \\
\hline & PLC3 & AT5G58670 & 5 & TCCATCACCAGAAGAACTTAAGGG & CAACATGAGGATCGTAGTTTGACG \\
\hline & & & & CAGAGAATGGAGTTATGACCGTTG & TGCGATATAGGAGCATCCATGTC \\
\hline & & & & CCATGATTACTCTCAAGACGACAGG & AGCACAACAGGATAAGCAGACACC \\
\hline & PLC4 & AT5G58680 & 5 & CTTTGGCTTGAAGATCTGGAGTC & TTGTAGAGTATACCGAGGGCAGG \\
\hline & PLC5 & AT5G58690 & 5 & AAGGAAGTTCAGGATGACGGAGT & CAGCTGCAGAGTACACACCAAGA \\
\hline & PLC6 & AT1G64050 & 1 & GGCTAAGCTTATGGGATCTGAAGG & АTCTCTCTGTGTCCTCACCGTTTC \\
\hline & PLC7 & AT4G38690 & 4 & GGCTCTCACCTTTCCAAACAAC & CCTTGAAGTAGCTGGTTGCTGC \\
\hline & PLC8 & $\mathrm{T} 11 \mathrm{I} 11.160$ & 4 & ACCAACGATATCGGAATACCTCTG & ATCTGCAACTTATCTCCACATCCC \\
\hline & PLC9 & $\mathrm{T} 11 \mathrm{I} 11.170$ & 4 & СССАTCTCTCTGTGGATAAACTAACC & CGATCAGTAACCTGTTTAACCCAC \\
\hline & PLC10 & AT4G17050 & 4 & GTATACGGCGAAAGAAAGCCAT & TCGTCTTCTCATCCGATTCAAC \\
\hline & PLC11 & AT4G34920 & 4 & GGCTGGTCTTACGTTGGAGAAAC & ATCCGATCAGTCACCGGTTTAAC \\
\hline & PLC12 & AT4G34930 & 4 & GAAGTGGTAAATCCAACGGTGTC & TCTCTGATACCGGCTTGTTGAAC \\
\hline & PLC13 & AT4G38530 & 4 & TGTGAGTGAAGTCCAAGGAGAAC & GGATAGTCAGACACATGAAACGC \\
\hline & PLC14 & AT4G38690 & 4 & GCTCTCACCTTTCCAAACAACTC & TTCCTTGAAGTAGCTGGTTGCTG \\
\hline & PLC15 & AT3G55940 & 3 & GTCGAAGCAAACATACAAAGTCTG & GCTCCACAGGTGATGTAAGAGTC \\
\hline & PLC16 & DL4555W & 4 & CTCAGATTACGTGTAGAAGACGG & GTGAAGGAGAGAGTGTTGGATTC \\
\hline & PLC17 & AT2G26870 & 2 & TGATTCAACTACTATCCGTCACG & CTTGTAGACAGGAACCTTATCAGG \\
\hline \multirow[t]{6}{*}{ PLD } & PLD $\alpha-1$ & AT3G15730 & 3 & AAGCTAGAGTTGGGAGAACCAGG & CTTTGGACTATGCTACCACCGTC \\
\hline & PLD $\alpha-2$ & AT1G52570 & 1 & AAGTGCTAAGTTTCCAGGAGTTCC & AAGCAATAGGTCCTTCAAGACGAC \\
\hline & PLD $\alpha-4$ & AT1G55180 & 1 & CTATCACCATCACGCTCAAGACTC & CACCCGTGTATCCAGTGTTATTG \\
\hline & PLD $\beta-1$ & AT2G42010 & 2 & ACССТTATTACССТTACAGGCСТC & GAATCGACAATGATAGAGTTGCCC \\
\hline & $\operatorname{PLD} \beta-2$ & AT4G00240 & 4 & GGGTGATATGTTTGGAAGATTGC & GATGCAGGACCTAATTTATCACG \\
\hline & PLD $\gamma-1$ & AT4G11850 & 4 & ACССТTATTACССТTACAGGCСТC & GAATCGACAATGATAGAGTTGCCC \\
\hline
\end{tabular}




\begin{tabular}{|c|c|c|c|c|c|}
\hline & Names & Gene ID & Chr. & Forward primer $\left(5^{\prime}-3{ }^{\prime}\right)$ & Reverse primer $\left(5^{\prime}-3^{\prime}\right)$ \\
\hline & \multirow[t]{2}{*}{ PLD $\gamma-2$} & \multirow[t]{2}{*}{ AT4G11830 } & \multirow[t]{2}{*}{4} & CTTGGGACTTTGATCTTAGGAGC & GCTGGTGCTGTCATAGGAAGAAC \\
\hline & & & & CATCCGGCTTATACTGAGACTATG & GATACAGAGTAACCCTACCGCC \\
\hline & \multirow[t]{2}{*}{ PLD $\gamma-3$} & \multirow[t]{2}{*}{ AT4G11840 } & \multirow[t]{2}{*}{4} & CATCCGGCTTATACTGAGACTATG & TCCTGATACAGAGTAACCCTACCG \\
\hline & & & & GACCAGCAACTCGTTCCGTTAG & TCGGAAGAGTACCGTCATCGAC \\
\hline & \multirow[t]{2}{*}{$\operatorname{PLD} \delta$} & \multirow[t]{2}{*}{ AT4G35790 } & \multirow[t]{2}{*}{4} & AGTTAGGTCGGAGGATTCGTAAAG & CСАACАСТСТААСАССТТСТTGAG \\
\hline & & & & GACTATGTCTATGGGAGGAGGG & AAGAGTAACCCTACCGCCTTTC \\
\hline & \multirow[t]{2}{*}{ PLD $\xi-2$} & \multirow[t]{2}{*}{ AT3G05630 } & \multirow[t]{2}{*}{3} & GACTATGTCTATGGGAGGAGGGTC & CTGATAAAGAGTAACCCTACCGCC \\
\hline & & & & CATGGATCGTCCTCTGGAATTATC & AGGCTCTTTCAACATGTGGGTATC \\
\hline \multirow{7}{*}{ PLA2 } & \multirow{2}{*}{ PLA2-1 } & \multirow{2}{*}{ AT1G61850 } & \multirow[t]{2}{*}{1} & GGAGAAAGTATCGGAGGACGTTA & TAACATGAGCGTCCTGGTAAAGC \\
\hline & & & & AGAATGACCAGAGCTGATGCTG & TGTTCTTGCTTGTCGTGAAGTT \\
\hline & \multirow[t]{2}{*}{ PLA2-2 } & \multirow[t]{2}{*}{ AT3G45880 } & \multirow[t]{2}{*}{3} & TCTACATGTTCTTCTCCATCCGC & TAAGATGCGATAACGGTCACCTG \\
\hline & & & & GGAGAGAAGTGAGAGAATTGAGC & ATAGAGACGGTGAACATCAGTCG \\
\hline & PLA2-3 & AT4G29460 & 4 & TGCTTTGTCACGTGTTGCTTTC & ACCTGCGCAGAGATTATTCACA \\
\hline & PLA2-4 & AT4G29470 & 4 & CCTATCTCTGTTTCAGTTCTCGG & GCACTTGCTGGCTTCTTAGGTA \\
\hline & PLA2-5 & AT4G29780 & 4 & TCAGGACCGAGTCATAGGAGGTTA & CTATCCAGCTGTCACGTAACATCC \\
\hline \multirow[t]{9}{*}{ PL } & PL1 & AT2G39400 & 2 & TCATTACTCTGCAGCTGGTGAAAC & ATCGAGTACGACGTCTTCTGATTC \\
\hline & PL2 & AT2G39410 & 2 & GTTGAACTCACGAGGCATGAAGT & TCTGGCTAAGAGCAACACAACTG \\
\hline & \multirow[t]{2}{*}{ PL3 } & \multirow[t]{2}{*}{ AT2G39420 } & 2 & GTTGTTCACATGCAGATGGCTAC & СТССТСССАТTGACTCТССТАAC \\
\hline & & & & TGCAGGCAAGTGAAACAGAGAAC & TTTGGGACATAAGCACTAAGCCC \\
\hline & PL4 & AT2G47630 & 2 & TTTAGAATGCGGAATCAGGCTG & TTGACGAGGTCCTTGAAAGCAG \\
\hline & PL5 & AT3G03540 & 3 & AAAGGCTCAGAATCGTATCCGA & AACCAATTCCTTGTAAACCGGC \\
\hline & PL6 & F27M3_29 & 1 & GATGAAGAATCTCCTCCAACTCC & TTCTCTGATAGCAGTTGTGCGAC \\
\hline & PL7 & AT4G38550 & 4 & CGCAGAGACCACTACGACATGTA & GGAGAGCTGTTTCGTGGTGTTAC \\
\hline & PL8 & AT4G38560 & 4 & GACGTTGTCAGTCTACCTCTACATTC & GAGTTGTGTGTTACGTTCAATCG \\
\hline IPK & IPK1 & AT5G07370 & 5 & TGTAGCAAGGAAACGCCTCTTAC & GTTACCACCGATGTTGAAGGATG \\
\hline & IPK2 & AT4G08170 & 4 & GGTTGCAGGACACATTGCTAAAG & GGTCTAGCATCATCATCATTTCCC \\
\hline & & & & TGAAGCTAACGGACAACGAAGAG & ACAATATCAAACGGACCCTGCTC \\
\hline & IPK3 & AT5G16760 & 5 & TACTTAGTTGGATACGCACTCGC & TGTTCTTGTCCTCTTGAGCAGTC \\
\hline & IPK4 & AT4G33770 & 4 & CTTTGGAGGTAGCTCAGCAACAC & GACAATCGATGCTTCAGGGTATG \\
\hline PI3K & PI3K1 & AT1G60490 & 1 & AGTTTCGTTTCTTCCTCTCCTGC & CCAGGAGTAGAGGTTGGAAATGA \\
\hline IPR & IPR1 & AT3G10380 & 3 & GTCAAAGTCCGCGTGTAGTCAGG & TGTAGCCTCCAGTAGCTTGGGAT \\
\hline & IPR2 & AT5G27230 & 5 & TTGATTTGGAACAGAAGGCAGAG & GTCATTCTTCATATTGGCTTTAGC \\
\hline IPPase & IPPase 1 & AT1G65880 & 1 & AATGGAAGATCGTCAAAACG & ATACAAACAACAGCAGCAGACCC \\
\hline & IPPase 2 & AT4G18010 & 4 & ATGGAAGATCGTCAAAACGATCA & CATAAGCCGAAATGGCAATGGA \\
\hline & IPPase3 & AT2G43900 & 2 & AGAAGTGGTTCCATTAAACGCTG & ATCTTGTCACCATCCTCTTCGTC \\
\hline & IPPase 4 & AT2G32010 & 2 & AACAACCACCGTGACGAAAA & TCATCACCATCATCAAGCACA \\
\hline & & & & GCTGGTAATGTTCTTGGAGC & GACGTGTTCTCGTAACTCG \\
\hline & IPPase5 & AT2G31830 & 2 & CGTTGGCATCGCTTGTCCCTGC & CATCTGCAGTAGTCGTATTGGG \\
\hline & & & & CGCAAATATAGCCTTGATGAGGG & CCATGAGAGTCGTTCCTTGAAAG \\
\hline & IPPase6 & AT1G05630 & 1 & CGAAGAAGAAGACGAAGAGGCG & GGAGACACGCTCCTTGAAAGG \\
\hline & & & & GCATTCCTATAGCCTACAATTCG & AGAGCTCTGATCCATCTTCCAAG \\
\hline & IPPase8 & AT2G01900 & 2 & GCCGATTTCCCACCTAATACTGA & AGATCCTCCATGTCAAGTCCATC \\
\hline & IPPase9 & AT1G71710 & 1 & GAGTTATAGTGATCCACCATCTCC & CAACACCAACAGTAGAGACTCGT \\
\hline & & & & GAGCTGAAGATGATCGTCCTGTT & TCСТCTCCATTAGCAACTTCAGG \\
\hline & IPPase 10 & AT1G34120 & 1 & GAAATCGTTCCCTTAAATGCTGG & ACAAAGGTTGCTTATGTGCTTGC \\
\hline & IPPase11 & AT1G71710 & 1 & TTCTGGAGGAAGACTTAGCCCAA & ACATCTAAGTTTCTGCGGCGTTC \\
\hline & IPPase12 & AT5G65090 & 5 & TAGGCCTATGGCACCAACTACAG & TGGAATCAACTCTCTTCTAGCCC \\
\hline
\end{tabular}


Tab. 2 The correlation coefficient of ratios from two replicates in each experiment. /WT: treated seedling versus untreated seedling. KT: 6-furfurylamino-purin (kinetin), one type of synthesized cytokinin. BR: 24-eBL. Drought ${ }^{\mathrm{a}}$ : mixed reverse PCR primers were used in reverse transcription; Drought ${ }^{\mathrm{b}}$ : oligo-dT primers were used for comparison of antisense primer data as an additional reproducibility control.

\begin{tabular}{|c|c|}
\hline & $\begin{array}{l}\text { Correlation } \\
\text { Coefficient }\end{array}$ \\
\hline Leaf-Cy5 versus flower-Cy3 & 0.93 \\
\hline Leaf-Cy5 versus stem-Cy3 & 0.92 \\
\hline $\mathrm{KT} / \mathrm{WT}$ & 0.98 \\
\hline IAA / WT & 0.95 \\
\hline $\mathrm{BR} / \mathrm{WT}$ & 0.78 \\
\hline $\mathrm{ABA} / \mathrm{WT}$ & 0.97 \\
\hline $\mathrm{GA}_{3} / \mathrm{WT}$ & 0.81 \\
\hline Drought ${ }^{\mathrm{a}} / \mathrm{WT}$ & 0.97 \\
\hline Drought $^{\mathrm{b}} / \mathrm{WT}$ & 0.89 \\
\hline $\mathrm{SA} / \mathrm{WT}$ & 0.94 \\
\hline Cold / WT & 0.99 \\
\hline MeJA / WT & 0.84 \\
\hline $\mathrm{CaCl}_{2} / \mathrm{WT}$ & 0.89 \\
\hline Mannitol / WT & 0.86 \\
\hline $\mathrm{NaCl} / \mathrm{WT}$ & 0.83 \\
\hline
\end{tabular}

PCR amplified DNA products were used for DNA chip preparation. Primers were based on the isolated and annotated mRNA sequences and were designed to amplify Nterminal fragments within the first few exons, so as to avoid nonspecific amplification. 93 PCR fragments, representing 79 independent clones, were amplified and purified for chip preparation. Repetition of the clones was due to repetition within the database, i.e. the genomic and mRNA sequences of one gene being assigned different accession numbers. These were carefully analyzed and compared after hybridization, to increase data veracity.

DNA fragments were spotted four times on each chip to increase the power of the experiment by showing reproducibility. A. thaliana total RNA was extracted from different treated and untreated tissues, fluorescently labeled via reverse transcription and then used for hybridization. The resulting images were scanned and analyzed. To confirm the reproducibility of the results, each hybridization was repeated at least four times, once with the $\mathrm{Cy} 3 / \mathrm{Cy} 5$ probe labeling reversed. As shown in Fig 1A and Tab 2, the signals were very similar across the replicates; the correlation coefficient for most of the experiments was around 0.9 , indicating that the results were reproducible. As an additional control, reverse transcription was performed with both oligo-dT primers and a mixture of the reverse primers used for PCR amplification in the drought treatment experiments, revealing the reproducibility of the results in a second fashion (Tab 2). And finally, we note
A

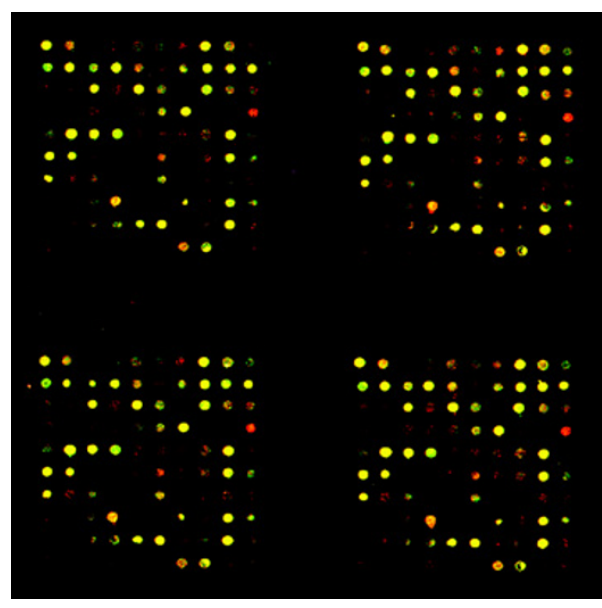

B

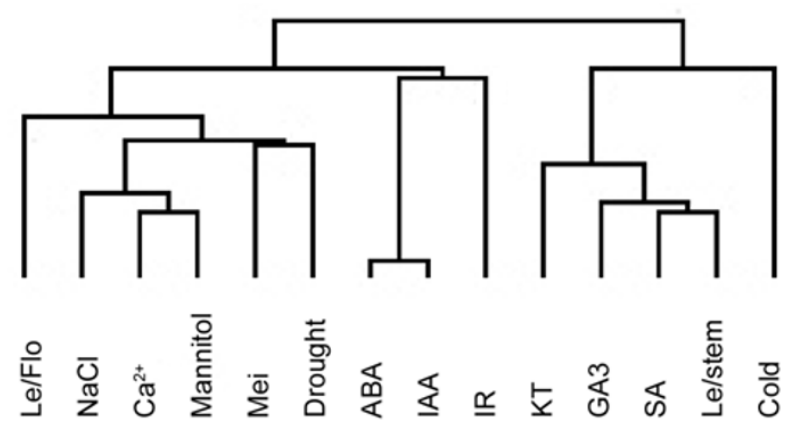

Fig 1. Analysis of PI pathway-related genes via DNA chip. (A) A typical image after hybridization using drought-treated materials. DNA samples were spotted on the glass slide in quadruplicate to assess the reproducibility of results. (B) Cluster analysis of the hybridizations.

that our experiments were in good agreement with the differential expression of previously studied genes including PI4K $\beta 1$ (AB008266) treated with auxin (IAA) and $\mathrm{NaCl}$ [7] and AtPLC1 (D38544) and AtIP5PII (IPPase2, AF289634) treated with ABA and drought $[35,36]$.

\section{Differential expression patterns of PI-involved genes}

Expression patterns of PI-involved genes in the vegetative tissues including leaf, stem and roots, and hormonetreated and stressed tissues were studied. Cluster analysis (http://rana.stanford.edu/software) showed that expression profilings of PI pathway genes was similar following treatments with ABA and IAA, $\mathrm{Ca}^{2+}$ and mannitol (Fig 1B).

PIS, using CDP-DG and inositol as substrates, generates PI which is not only the starting point of PI pathway but also the major component of membrane. Three members of the PIS gene family (one cloned and two putative ones) were identified and all of them were expressed in all tissues tested including leaf, root stem and flowers. Nearly constitutive expression was seen following hormone treat- 
Tab 3. Differential expressions of PI involved genes. Numbers show the ratios sample vs. control after hybridization. Clones with ratios $>2$ or $<0$. 5 were selected, which indicated at least one time induction or suppressions. To expand the evaluations, clones with ratios $>1.5$ or $<0.75$ were supplemented. Le, leaf; Fl, flower; St, stem; Man, mannitol [for detailed information please see "materials and methods", plants were treated with IAA $(10 \mu M)$, 24-epi-brassinolide (24-eBL, $10 \mu M)$, ABA (10 $\mu M)$, KT (10 $\mu M)$, GA3 (10 $\mu M)$, SA (salicylic acid, 200 $\mu M$ ), MeJA (Methyl Jasmonate, $100 \mu \mathrm{M})$, mannitol $(250 \mu M), \mathrm{CaCl}_{2}(5 \mathrm{mM})$ or $\mathrm{NaCl}(200 \mathrm{~m} M)$ for $4 \mathrm{~h}$, and with cold $\left(4^{\circ} \mathrm{C}\right.$, for $\left.16 \mathrm{~h}\right)$ or drought $\left(55^{\circ} \mathrm{C}\right.$, for $\left.\left.1 \mathrm{~h}\right)\right]$.

\begin{tabular}{|c|c|c|c|c|c|c|c|c|c|c|c|c|c|c|}
\hline Names & $\begin{array}{c}1 \\
\text { Le/Fl. }\end{array}$ & $\begin{array}{c}2 \\
\text { Le/St. }\end{array}$ & $\begin{array}{c}3 \\
\text { IAA } \\
\end{array}$ & $\begin{array}{c}4 \\
\text { BR } \\
\end{array}$ & $\begin{array}{r}5 \\
\text { KT } \\
\end{array}$ & $\begin{array}{c}6 \\
\text { ABA }\end{array}$ & $\begin{array}{r}7 \\
\mathrm{GA}_{3} \\
\end{array}$ & $\begin{array}{c}8 \\
\text { Dry } \\
\end{array}$ & $\begin{array}{c}9 \\
\text { Man. }\end{array}$ & $\begin{array}{c}10 \\
\mathrm{NaCl} \\
\end{array}$ & $\begin{array}{c}11 \\
\mathrm{Ca}^{2+} \\
\end{array}$ & $\begin{array}{r}12 \\
\mathrm{SA} \\
\end{array}$ & $\begin{array}{c}13 \\
\text { MeJA } \\
\end{array}$ & $\begin{array}{c}14 \\
\text { Cold } \\
\end{array}$ \\
\hline IP3K 1 & & 4.161 & 1.938 & & & 2.078 & 1.518 & & & & & 1.738 & & \\
\hline IP3K3 & 2.309 & 0.287 & 1.518 & & 0.579 & 1.627 & & 1.599 & 1.868 & & & & 1.650 & \\
\hline IP3K4 & & 1.875 & & & & & & & & & & 2.295 & & \\
\hline PI3K 1 & & & & & & & & & 2.020 & & & & 0.747 & \\
\hline IPR1 & 0.650 & & 2.573 & 2.160 & & 1.518 & 0.445 & & & 1.625 & & & & 1.808 \\
\hline IPR2 & & 2.942 & & 1.749 & & & & & & & & & & \\
\hline PIS1 & 1.625 & 10.552 & 1.540 & 0.544 & & 1.918 & 1.562 & & & & & 4.396 & & \\
\hline PIS2 & & & & & & & 1.612 & & & & 0.653 & 1.583 & & 0.585 \\
\hline PIS3 & & 2.525 & 1.664 & 1.521 & & & & & & & & & & \\
\hline PI4K $\beta 1$ & & & 4.301 & & & & 0.720 & 0.673 & & 1.891 & & 0.400 & & \\
\hline $\mathrm{PI} 4 \mathrm{~K} \alpha 2$ & & & & & 0.470 & & & & & & & & & 0.460 \\
\hline PI4K $\beta 2$ & & 2.061 & & & 0.731 & & & 3.563 & & & & & & 0.663 \\
\hline PIPK1 & 0.704 & & & & & & & & & & 0.687 & & & \\
\hline PIPK2 & & & & & & & & & & 0.647 & & 1.591 & & \\
\hline PIPK4 & & & 1.525 & & & & 2.776 & 0.638 & & & & 2.770 & & 1.882 \\
\hline PIPK5 & 3.771 & 2.468 & & & & & & & & & & & & \\
\hline PIPK6 & & 0.514 & & & 3.983 & & 1.817 & & & 0.651 & 0.730 & & & \\
\hline PIPK7 & & 5.307 & 0.500 & 0.717 & & 0.734 & & & & & & 1.996 & & 0.325 \\
\hline PIPK8 & & & & & & & & 0.532 & & 1.611 & & & & \\
\hline PIPK9 & & & & & & & 2.265 & & & & & & & 0.671 \\
\hline PIPK 10 & 1.602 & & & & & & & 2.133 & 1.587 & & 0.617 & & & \\
\hline PLA2-1 & & & & & & & & & & & & & & 0.345 \\
\hline PLA2-2 & & & & & 0.744 & & 1.642 & & & & & 0.592 & & \\
\hline PLA2-4 & & 1.747 & & 0.689 & 1.935 & & & & & & & 0.534 & & \\
\hline PLA2-5 & 0.058 & & 0.243 & & & 0.586 & & 0.265 & 0.029 & 0.318 & 0.065 & 2.044 & 0.574 & 16.846 \\
\hline PL1 & 0.333 & 0.107 & & 0.680 & 0.199 & 0.656 & 0.424 & 1.636 & 1.655 & & & 0.220 & 0.565 & \\
\hline PL2 & & 3.072 & 0.368 & & 1.526 & & 2.080 & & & & & 1.919 & & 0.576 \\
\hline PL3 & 0.635 & & 0.748 & & & & & 6.437 & 1.645 & & & & 2.371 & 0.696 \\
\hline PL4 & & 1.797 & & 1.896 & & & & 1.886 & & & 0.531 & & 1.527 & \\
\hline PL5 & 2.124 & 2.251 & & & & & & & & & & & & \\
\hline PL6 & & & & 1.732 & 0.685 & & & 0.609 & 1.531 & & & & & 0.489 \\
\hline PL7 & 0.447 & & 1.500 & & 3.008 & & 0.706 & 0.673 & & & & 0.483 & 0.709 & \\
\hline PL8 & 0.368 & & & & & & & & 0.680 & & 0.524 & 0.665 & 0.430 & \\
\hline
\end{tabular}




\begin{tabular}{|c|c|c|c|c|c|c|c|c|c|c|c|c|c|c|}
\hline Names & $\begin{array}{c}1 \\
\text { Le/FI. }\end{array}$ & $\begin{array}{c}2 \\
\text { Le/St. }\end{array}$ & $\begin{array}{c}3 \\
\text { IAA }\end{array}$ & $\begin{array}{c}4 \\
\text { BR }\end{array}$ & $\begin{array}{c}5 \\
\text { KT }\end{array}$ & $\begin{array}{c}6 \\
\mathrm{ABA}\end{array}$ & $\begin{array}{c}7 \\
\mathrm{GA}_{3}\end{array}$ & $\begin{array}{c}8 \\
\text { Dry }\end{array}$ & $\begin{array}{c}9 \\
\text { Man. }\end{array}$ & $\begin{array}{c}10 \\
\mathrm{NaCl}\end{array}$ & $\begin{array}{c}11 \\
\mathrm{Ca}^{2+}\end{array}$ & $\begin{array}{l}12 \\
\text { SA }\end{array}$ & $\begin{array}{c}13 \\
\text { MeJA }\end{array}$ & $\begin{array}{c}14 \\
\text { Cold }\end{array}$ \\
\hline AtPLC1 & & 2.684 & 1.713 & 2.154 & 1.942 & 1.562 & & 2.623 & & & & & 1.628 & 0.714 \\
\hline PLC2 & & 0.252 & & 1.641 & 0.395 & & 0.365 & 2.009 & & & & 0.474 & & 0.638 \\
\hline PLC3 & & & 1.601 & & & & 2.253 & 2.556 & & & 0.661 & & 2.184 & \\
\hline PLC4 & 2.217 & 0.391 & & & & & 0.510 & & & & & 0.410 & & \\
\hline PLC5 & & 2.588 & 0.004 & & & 0.003 & & & & & & & & \\
\hline PLC6 & & 3.370 & & & 2.038 & & 0.526 & 0.656 & & 1.514 & & & & \\
\hline PLC7 & 0.476 & 2.246 & 1.647 & 0.630 & 2.056 & 1.503 & 1.552 & 0.499 & & & & & 0.748 & \\
\hline PLC8 & 1.913 & & 1.940 & & & 1.774 & & 0.524 & 2.781 & 2.657 & 2.980 & & 1.989 & \\
\hline PLC9 & 2.195 & 0.734 & 1.501 & & 0.631 & & & 0.500 & 1.976 & 2.021 & & & 1.712 & \\
\hline PLC10 & & & & & 0.578 & & & & & & & 1.724 & & \\
\hline PLC11 & 2.198 & 0.563 & & & & & & & 1.610 & 1.555 & & 0.467 & & \\
\hline PLC12 & & 2.038 & & & & & & & & & & 2.712 & & \\
\hline PLC13 & 3.090 & 2.678 & 0.665 & 1.556 & & & 0.749 & & & & 0.728 & 0.669 & & 0.622 \\
\hline PLC14 & 0.738 & 0.255 & & & 0.444 & & 0.722 & 1.724 & 2.045 & & 1.761 & 0.618 & & \\
\hline PLC15 & & 0.174 & 0.341 & 0.679 & 0.334 & & 0.464 & & & & & 0.453 & & 0.391 \\
\hline PLC16 & & & & & 0.559 & & & & & & & & & 0.621 \\
\hline PLC17 & 0.687 & & & & & & 0.717 & 0.626 & & & & 0.430 & 0.671 & \\
\hline PLD $\alpha$ & 0.665 & & 1.815 & & & & 0.647 & & & & & & 0.742 & \\
\hline PLD- $\alpha 2$ & & & & & & 0.625 & 2.620 & 0.616 & & & & & & \\
\hline PLD- $\alpha 4$ & & & & & & & & 0.734 & & 0.584 & 0.386 & & & 0.347 \\
\hline PLD- $\beta 1$ & & & 0.754 & 0.455 & & 0.455 & & & & & & 0.545 & & \\
\hline PLD- $\beta 2$ & & 4.043 & & 0.584 & 3.299 & & 1.671 & & & & 0.628 & & 0.632 & \\
\hline PLD- $\gamma 1$ & 0.402 & 2.317 & & 2.636 & 0.664 & & & & & & & & & 1.761 \\
\hline PLD- $\gamma 2$ & 0.632 & 1.807 & & 1.789 & & & 0.704 & & & & & & & \\
\hline PLD- $\gamma 3$ & & 1.966 & 1.673 & 1.588 & & & & & & & & & & \\
\hline PLD- $\delta$ & & 0.410 & 2.000 & & 0.556 & & 0.668 & & & 1.548 & & 0.712 & & \\
\hline PLD $\xi-2$ & 1.581 & 0.450 & & & 0.445 & & 0.406 & 1.783 & & & & 0.516 & & \\
\hline IPPase1 & 0.231 & 2.183 & 0.644 & & & & & & 0.384 & 0.625 & 0.612 & 0.732 & & \\
\hline IPPase 2 & 1.700 & 2.038 & 1.551 & 0.614 & & 1.578 & & 1.755 & 0.709 & & 0.712 & & & \\
\hline IPPase3 & & & & & & & 4.175 & 1.687 & & 1.653 & & & & \\
\hline IPPase4 & 1.776 & & 1.990 & & & 1.592 & & 0.322 & 1.605 & & & & & 2.397 \\
\hline IPPase 5 & & 3.865 & & 0.534 & 1.818 & & & & & 1.882 & & & & \\
\hline IPPase6 & & & & & & & & & 0.466 & & 0.545 & & 0.583 & \\
\hline IPPase8 & & 0.207 & 0.451 & & 0.301 & & 0.559 & & & & & 0.444 & & 0.439 \\
\hline IPPase9 & 1.540 & & & & 2.220 & & & & & 0.740 & 0.507 & & 0.691 & 2.214 \\
\hline IPPase 10 & & & & & 2.230 & & & & & 0.655 & & & & \\
\hline IPPase11 & & & & & & & 2.268 & & 2.038 & & 0.609 & & & 0.562 \\
\hline IPPase 12 & & & 0.739 & & & & & & & 1.686 & & & & \\
\hline
\end{tabular}


ment and environmental stresses except for the expression of PIS1 following SA treatment (Tab 3), which increased significantly.

Phosphorylation of PI at 4-position, by PI4K, generates PI4P involving in reorganization of cell skeletons, and so on. Analysis on A. thaliana genome identified four members of the family (two known, two putative) and studies via DNA chips indicated that expression of PI4K $\alpha 2$ was relatively high in leaves though it did not change following hormone treatment or stress, while PI4K $\beta 1$ and PI4K $\beta 2$ were present in the normal tissues and up-regulated by auxin and down-regulated by cytokinin, respectively. Additionally, expressions of both were regulated following SA treatment and cold stress (Tab 3), in a manner similar to that seen in rice $O s P I 4 K 1$ [8], which was induced by SA, suggesting the involvement of PI4K in plant wounding responses.

The presence of more than 10 isoforms of PIPK in the A. thaliana genome suggested that it could be differentially expressed in different tissues, or during cellular responses to environmental factors such as, which has been demonstrated, $\mathrm{NaCl}$, drought or ABA. As shown in Tab 3 besides the constitutive expression in various tissues, some PIPKs were differentially expressed, i.e. relatively highly expressed in leaves (PIPK5, 7), up-regulated by cytokinin (PIPK6), GA 3 (PIPK4, 9), mannitol (PIPK10) and SA treatment (PIPK4, 7), or down-regulated following auxin treatment or cold stress (PIPK7) (Tab 3).

Phospholipases, including PLC, PLD and PLA $\mathrm{P}_{2}$, are believed to play significant roles in multiple developmental stages and constitute the largest gene family of PI pathway identified in the $A$. thaliana genome. The identified phospholipases belonged to the PLC (17 members), PLD (12 members), $\mathrm{PLA}_{2}$ (4 members), or putative phospholipase ( 8 members) families. Most PLCs were highly expressed in leaves (4-9, 11-13), but fewer were expressed in flowers (PLC 7) and stems (PLC2, 4, 14, 15). Most PLDs were constitutively expressed in all tested tissues, except for PLD $\beta-2$ (higher in leaves) and PLD $\xi-2$ (higher in leaves and stems) (Fig 3, Tab 3). Putative PL2 and 5 were highly expressed in leaves, while PL1 was strongly expressed in flowers (PL7 and 8) and stems (Fig 3, Tab 3).

With the focus on the expression profiling under hormone and stresses conditions, as revealed by DNA chips, PLCs were differentially expressed in response to auxin (PLC8 was up- and PLC5 and 15 were down-regulated),

-Fig 2. Cluster analysis of PI-involved genes in flowers (A) and stems (B), and following hormone treatment (C) or environmental stresses (D).
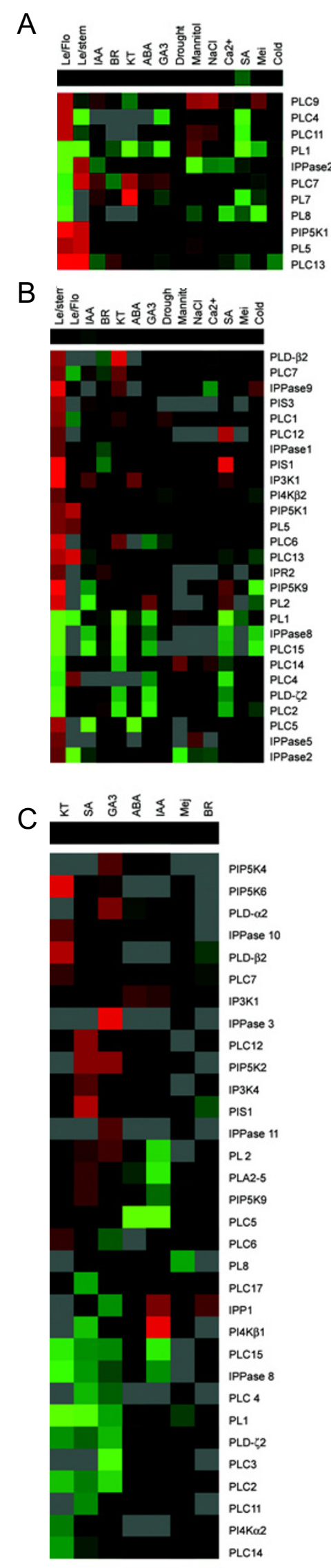

D

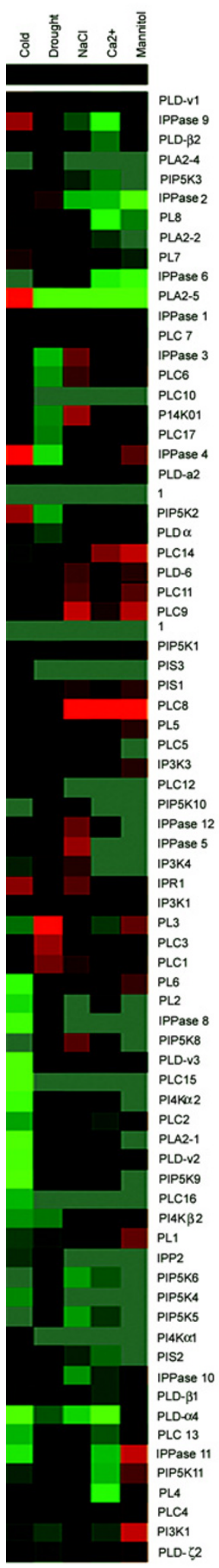




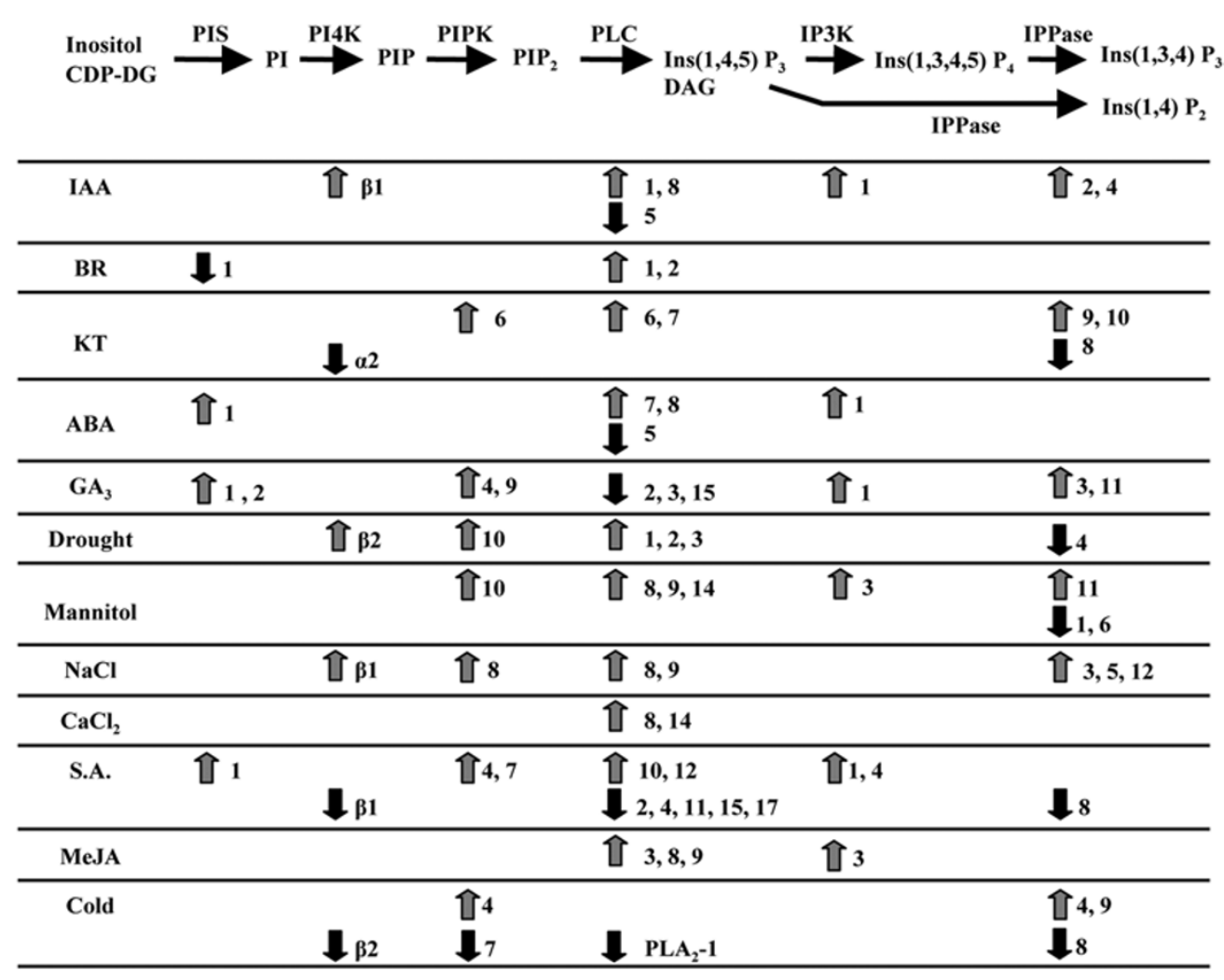

Fig. 3 Draft model of involvement of PI pathway-related genes in multiple plant developmental processes. Multi-gene family of PI pathway, as well as individual isoform, is multi-functionally (with distinguished alterative trend) involved in plant developmental processes and cell responses to environmental factors, making up of a complex network.

cytokinin (AtPLC1, PLC6 and 7 were up- and PLC2, 14 and 15 were down-regulated), ABA (AtPLC1 was up- and PLC5 was down-regulated) and $\mathrm{GA}_{3}$ (PLC2-4 and 15 were down-regulated) (Fig 3), as well as cell response to drought (PLC1), mannitol (PLC8, 9 and 14), $\mathrm{NaCl}$ (PLC8 and 9), $\mathrm{Ca}^{2+}$ (PLC8), SA (PLC12 up, PLC2, 4, 11, 15 and 17 down), MeJA (PLC8) and cold treatment (PLC15 down). PLDs were altered in response to plant hormones including brassinosteroid (PLD $\gamma-1)$, and $\mathrm{GA}_{3}(\mathrm{PLD} \alpha-2)$. Only PLD $\alpha-4$ was suppressed by cold treatment. PLA ${ }_{2}$ has been reported to be regulated by auxin. In addition, $\mathrm{PLA}_{2}-1$ and 4 were suppressed by cold and SA treatment, and $\mathrm{PLA}_{2}-5$ was suppressed by drought, mannitol, $\mathrm{NaCl}$ and $\mathrm{Ca}^{2+}$, and up regulated by SA and cold. Several putative phospholipases were transcribed in leaves, flowers and stems, and were suppressed by cytokinin (PL1), GA 3 (PL1), SA (PL1 and 7), MeJA (PL8) and cold (PL6), or up-regulated by cytokinin (PL7) or SA (PL2).

A few members of the IPK, PI3K and IPR families were identified. These were constitutively transcribed in many of the normal and treated tissues, though some were regulated by auxin (IPK1, IPR1), brassinosteroid (IPR1), ABA (IPK1), SA (IPK4) or mannitol (PI3K1). Fifteen isoforms were classified as IPPases based on homologous analysis, and 12 of these were analyzed by DNA chip technology. IPPase 1, 2 and 5 were highly expressed, while expression of IPPase 8 was lower in stems, and IPPase 2 was lower in flowers. Further analysis revealed that some IPPases were up regulated by plant hormones including auxin (IPPase4), cytokinin (IPPase9, 10), $\mathrm{GA}_{3}$ (IPPase3 and 11), while IPPase8 was down regulated by both auxin and cytokinin. IPPase 1 and 6 were suppressed by mannitol treatment while IPPase 11 was up regulated. IPPase 4 was up regulated under cold treatment, and IPPase6 was suppressed by mannitol treatment (Fig 3, Tab 3).

\section{DISCUSSION}

The PI metabolic pathway plays significant roles in many developmental processes, as well as in cell responses to environmental factors. Analysis of the expression patterns of genes involved in the pathway, particularly the individual isoforms, will aid in focusing various functional studies. Accordingly, we employed DNA chip technology for high-throughput analysis of the pathway, particularly the individual isoforms. A detailed analysis of the $A$. thaliana genome revealed the presence of 82 PI-involved clones, including 10 or more members of the PIPK, phospholipase and IPPase families. To ensure reliability and to 
minimize the inherent variability of microarray assays, several strategies were used. As replication is very important for array study[38], two double-dotted microarray slides (four replicates) were used to analyze the mRNA abundance of each sample pair in the studies. Also, the labeling of the sample in each pair was reversed on the second slide to overcome potential artifacts caused by dye-related differences in labeling efficiency, different laser settings, and nonlinearity of photomultiplier tubes in the scanner. In addition, in one experiment analyzing the expression profiles of drought-stressed seedlings, chips were labeled by probes reverse transcribed with different primers (commonly used oligo-dT vs. specific reverse PCR primers), which showed that the results were consistent across both methods. As illustrated in Tab 2, the correlation coefficient of the ratio from each replicate pair was $\sim 0.9$, confirming reproducibility among individual arrays in the same experiment. Therefore, signals from the replicates were reproducible and the conclusions derived from this analysis are reliable.

The presence of multiple members of PI pathway gene families within the genome suggests that there may be differential expression of individual isoforms in various tissues, during different developmental stages, or in response to environmental factors. Our results indeed showed differential expression of PI-involved genes in different tissues including stem, root and flowers (Fig 2A, B) and following treatment with various compounds or changes of temperature (Fig 2C, D). Differential expression patterns in stem, root and flowers suggest involvements of PI-related genes in the development of relevant tissues or cell types, which has been confirmed in root or root hairs (PIPK), filaments (PIPK and PLC), anthers (PLC and IPK), pollen grains and pollen tubes (IPK and IPPase).

As summarized in Fig 3, following hormone treatments and environmental stresses, both phospholipase and inositol polyphosphate phosphatase, both of which are capable of generating $\operatorname{Ins}(1,4,5) \mathrm{P}_{3}$ via hydrolysis or degrading Ins $(1,4,5) \mathrm{P}_{3}$ by dephosphorylation, were altered in almost all experimental cases. It has been shown that $\operatorname{Ins}(1,4,5) \mathrm{P}_{3}$ is capable of stimulating $\mathrm{Ca}^{2+}$ release from internal stores, resulting in the regulation of downstream $\mathrm{Ca}^{2+}$-dependent signaling pathways to involve the cell response to hormone, especially to stresses treatment including drought, $\mathrm{NaCl}$ and etc. However, involvement of different isoforms in various developmental processes indicates complicated regulating mechanisms. For example, IAA treatment, associated with plant growth, triggered up-regulation of members of the PLC and PIPK families. In other cases, PLC8 was up-regulated following IAA, ABA, mannitol, $\mathrm{NaCl}, \mathrm{Ca}^{2+}$ and MeJA treatments, while PLC5 was suppressed by IAA and ABA treatments, and isoforms of $\mathrm{PLA}_{2}$ were down-regulated in other situations, and different PLC isoforms were also altered. This was similar to the behavior of IPPase following KT and SA treatments, when IPPase 8 was suppressed whereas IPPase 9 and 10 were up-regulated, or the case of PIPK family members following treatment with $\mathrm{GA}_{3}$ and SA, when PIPK4, PIPK7 and 9 were up-regulated (Fig 2). Our detailed studies on the individual isoforms of PIPK and IPPase confirmed that these genes are differentially expressed under various conditions, suggesting that they have specific functions in plant hormone and stress responses.

Overall, chip technology facilitates the studies of expression profiles and physiological roles of the pathway. Differential expressions in different tissues or under treatment of environmental factors provide informative clues on functional characterization of relevant isoform. As many members of the pathway are not only precursors but also messenger molecules themselves, knowledge of their expressions will certainly help us to study the relevant signaling pathways. We believe the detailed analysis of the expression patterns presented herein is a good basis for further expression study and functional analyses of the differentially expressed genes.

\section{ACKNOWLEDGEMENTS}

This study was supported by grants from the National Natural Science Foundation of China (No. 30100101) and the State Key Project of Basic Research (No. G1999011604). We thank Dr. Yao Li for the preparation of the DNA chips and Ke Duan for help with data analysis.

Received, May 23, 2003

Revised, Oct 25, 2003

Accepted, Nov 23, 2003

\section{REFERENCES}

1 Munnik T, Irvine RF, Musgrave A. Phospholipid signaling in plants. Biochimica et Biophysica Acta 1998; 1389:222-72.

2 Berridge MJ, Bootman MD, Lipp P. Calcium-a life and death signal. Nature 1998; 395:645-8.

3 Carafoli, E. Calcium signaling: A tale for all seasons. Proc Natl Acad Sci USA. 2002; 99:1115-22.

4 Brown FD, Rozelle AL, Yin HL, Balla T, Donaldson JG. Phosphatidylinositol 4,5-biphosphate and arf6-regulated membrane traffic. J Cell Bio 2001; 154:1007-17.

5 Xue HW, Hosaka H, Mueller-Roeber B. Cloning of Arabidopsis thaliana phosphatidylinositol synthase and functional expression in the yeast pis mutant. Plant Mol Biol 2000; 42:757-64.

6 Stevenson JM, Perera IY, Boss WF. A phosphatidylinositol 4kinase pleckstrin homology domain that binds phosphatidylinositol 4-monophosphate. J Biol Chem 1998; 273: 22761-7.

7 Xue HW, Pical C, Brearley C, Elge S, Müller-Röber B. A plant 126-kDa phosphatidylinositol 4-kinase with a novel 
repeat structure: cloning and functional expression in baculovirusinfected insect cells. J Biol Chem 1999; 274:5738-45.

8 Kong XF, Xu ZH, Xue HW. Isolation and functional characterization of the C-terminus of rice phosphatidylinositol 4-kinase in vitro. Cell Research 2003; 13:131-9.

9 Anderson RA, Boronenkov IV, Doughman SD, Kunz J. Phosphatidylinositol phosphate kinases, a multifaceted family of signaling enzymes. J Bio Chem 1999; 274:9907-10.

10 Elge S, Brearley C, Xia HJ, Kehr J, Xue HW, Mueller-Roeber B. An Arabidopsis inositol phospholipid kinase strongly expressed in procambial cells: synthesis of $\operatorname{PtdIns}(4,5) \mathrm{P}_{2}$ and $\operatorname{PtdIns}(3,4$, 5) $\mathrm{P}_{3}$, in insect cells by 5-phosphorylation of precursors. Plant $\mathrm{J}$ 2001; 26:561-71.

11 Guo K, Nichol R, Skehel P, Dormann D, Weijer CJ, Williams JG, Pears C. Dictyostelium nuclear phosphatidylinositol phosphate kinase required for developmental gene expression. EMBO J 2001; 21:6017-27.

12 Westergren T, Dove SK, Sommain M, Pical C. AtPIP5K1, an Arabidopsis thaliana phosphatidylinositol phosphate kinase, synthesizes PtdIns $(3,4) \mathrm{P}_{2}$ and $\mathrm{PtdIns}(4,5) \mathrm{P}_{2}$ in vitro and is inhibited by phosphorylation. Bio J 2001; 359:583-9.

13 Barbieri MA, Heath CM, Peters EM, Wells A, Davis JN. Phosphatidylinositol-4 phosphate 5-kinase-1â is essential for epidermal growth factor receptor-mediated endocytosis. J Bio Chem 2001; 276:47212-6.

14 Sanchez JP, Chua NH. Arabidopsis PLC1 Is required for secondary responses to abscisic acid signals. The Plant Cell 2001; 13:1143-54.

15 Cocco L, Martelli AM, Gilmour RS, Rhee SG, Manzoli FA. Nuclear phospholipase $\mathrm{C}$ and signaling. Biochimica et Biophysica Acta $2001 ;$ 1530:1-14.

16 Wang XM. Multiple forms of phospholipase D in plants: the gene family, catalytic and regulatory properties, and cellular functions. Progress in Lipid Research 2000; 39:109-49.

17 Ishiguro S, Kawai-Oda A, Ueda J, Nishida I, Okada K. The defective in anther dehiscencel gene encodes a novel phospholipase A1 catalyzing the initial step of jasmonic acid biosynthesis, which synchronizes pollen maturation, anther dehiscence, and flower opening in arabidopsis. The Plant Cell 2001; 13:2191209.

18 Kim JY, Chung YS, Ok SH, Lee SG, Chung WI, Kim IY, Shin JS. Characterization of the full-length sequences of phospholipase A2 induced during flower development. Biochimica et Biophysica Acta 1999; 1489:389-92.

19 Valentin E, Lambeau G. Increasing molecular diversity of secreted phospholipases A2 and their receptors and binding proteins. Biochimica et Biophysica Acta 2000; 1488:59-70.

20 Grishin NV. Phosphatidylinositol phosphate kinase: A link between protein kinase and glutathione synthase folds. J of Molecular Bio 1999; 291:239-47.

21 Berdy SE, Kudla J, Gruissem W, Gillaspy GE. Molecular characterization of At5P1, an inositol phosphatase capable of terminating inositol trisphosphate signalling. Plant Physiol 2001; 126: 801-10.

22 Bertsch U, Deschermeier C, Fanick W, Girkontaite I, Hillemeier K, Johnen H, Weglhner W, Emmrichi F, Mayr GW. The second messenger binding site of inositol 1,4,5-trisphosphate 3-kinase is centered in the catalytic domain and related to the inositol trisphosphate receptor site. J Molecular Bio 2000; 275:115764.
23 Boronenkov IV, Loijens JC, Umeda M, Anderson RA. Phosphoinositide signaling pathways in nuclei are associated with nuclear speckles containing Pre-mRNA processing factors. Molecular Biology of the Cell 1998; 9:3547-60.

24 Erneux C, Govaerts C, Communi D, Pesesse X. The diversity and possible functions of the inositol polyphosphate 5-phosphatase Biochimica et Biophysica Acta 1998; 1436:185-99.

25 Ekblad L, Jergil B. Localization of phosphatidylinositol 4-kinase isoenzymes in rat liver plasma membrane domains. Biochimica et Biophysica Acta 2001; 1531:209-21.

26 Mueller-Roeber B, Pical C. Inositol phospholipid metabolism in Arabidopsis. Characterized and putative isoforms of inositol phospholipid kinase and phosphoinositide-specific phospholipase C. Plant Physiol 2002; 130:22-46.

27 Qin C, Wang XM. The Arabidopsis phospholipase D family. characterization of a calcium-independent and phosphatidycholine-selective PLD $\zeta 1$ with distinct regulatory domains. Plant Physiol 2002; 128:1057-68.

28 Meinke DW, Cherry JM, Dean C, Rounsley SD, Koornneef M. Arabidopsis thaliana: a model plant for genome analysis. Science 1998; 282:662-82.

29 Ma LG, Gao Y, Qu LJ, Chen ZL, Li J, Zhao H, Deng XW. Genomic evidence for COP1 as a repressor of light-regulated gene expression and development in arabidopsis. The Plant Cell 2002; 14:2383-98.

30 Girke T, Todd J, Ruuska S, White J, Benning C, Ohlrogge J. Microarray analysis of developing Arabidopsis seeds. Plant Physiol 2000; 124:1570-81.

31 Reymond P, Weber H, Damond M, Farmer EE. Differential gene expression in response to mechanical wounding and insect feeding in Arabidopsis. The Plant Cell 2000; 12:707-20.

32 Seki M, Narusaka M, Abe H, Kasuga M, Yamaguchi-Shinozaki K, Carninci P, Hayashizaki Y, Shinozaki K. Monitoring the expression pattern of 1300 Arabidopsis genes under drought and cold stresses by using a full-length cDNA microarray. The Plant Cell 2001; 13:61-72.

33 Lin F, Xu S, Ni WM, Chu ZQ, Xu ZH, Xue HW. Identification of rice $\mathrm{ABA}$-responsive genes in rice shoots via cDNA macroarray. Cell Research 2003; 13:59-68.

34 Perez-Amador MA, Lidder P, Johnson MA, Landgraf J, Wisman E, Green PJ. New molecular phenotypes in the $d s t$ mutants of Arabidopsis revealed by DNA microarray analysis. Plant Cell 2001; 13:2703-17.

35 Hirayama T, Ohto C, Mizoguchi T, Shinozaki K. A gene encoding a phosphatidylinositol-specific phospholipase $\mathrm{C}$ is induced by dehydration and salt stress in Arabidopsis thaliana. Proc Natl Acad Sci USA. 1995; 92:3903-7.

36 Mikami K, Katagiri T, Iuchi S, Yamaguchi-Shinozaki K, Shinozaki K. A gene encoding phosphatidylinositol-4-phosphate 5-kinase is induced by water stress and abscisic acid in Arabidopsis thaliana. Plant J 1998; 15:563-8.

37 Mueller-Roeber B and Pical C. Inositol Phospholipid Metabolism in Arabidopsis. Characterized and Putative Isoforms of Inositol Phospholipid Kinase and Phosphoinositide-Specific Phospholipase C. Plant Physiology 2002, 130:22-46.

38 Lee ML, Kuo FC, Whitmore GA, Sklar J. Importance of replication in microarray gene expression studies: Statistical methods and evidence from repetitive cDNA hybridizations. Proc Natl Acad Sci USA 2000; 97:9834-9. 